\title{
Intact learning and memory in rats following treatment with the dual orexin receptor antagonist almorexant
}

\author{
Hendrik Dietrich • François Jenck
}

Received: 17 November 2009 / Accepted: 23 June 2010/Published online: 15 July 2010

(C) The Author(s) 2010. This article is published with open access at Springerlink.com

\begin{abstract}
Rationale Orexins play a key role in the maintenance of alertness and are implicated in the modulation of diverse physiological processes, including cognitive function. Almorexant, a dual orexin receptor antagonist, transiently and reversibly blocks the action of orexin peptides at both $\mathrm{OX}_{1}$ and $\mathrm{OX}_{2}$ receptors and increases time spent in rapid eye movement (REM) and non-REM sleep.

Objectives We explored the direct effects on learning and memory of single and repeated administration of almorexant in rats.

Methods Following administration of high doses of almorexant (300 mg/kg, p.o.), scopolamine (0.8 mg/kg, i.p.), combination almorexant-scopolamine, or vehicle alone, rats were trained on a Morris water maze spatial navigation task, or on a passive avoidance task.

Results Rats treated with almorexant learned the spatial navigation task with similar efficacy as vehicle-treated animals. After 4 days, almorexant-but not vehicle-treated rats had established spatial memory; after 8 days, spatial memory had been established in both vehicle - and almorexant-treated rats. Scopolamine-treated rats failed to learn the spatial task. Both vehicle - and almorexant-but not scopolamine-treated rats demonstrated passive avoidance learning. Almorexant did not ameliorate scopolamineinduced impairment of learning in either task.

Conclusions Rats treated with almorexant are fully capable of spatial and avoidance learning.
\end{abstract}

This study was sponsored by Actelion Pharmaceuticals Ltd

\section{H. Dietrich $(\square) \cdot$ F. Jenck}

Research and Development, Actelion Pharmaceuticals Ltd,

Gewerbestrasse 16,

CH-4123 Allschwil, Switzerland

e-mail: hendrik.dietrich@actelion.com
Keywords Almorexant · ACT-078573 · Orexin · Rat ·

Morris water maze $\cdot$ Spatial learning .

Passive avoidance learning $\cdot$ Scopolamine

\section{Introduction}

The amount of time spent in rapid eye movement (REM) and non-REM (NREM) sleep exerts an influence on cognitive processes in animals and humans. Controversially debated (Genzel et al. 2009) and not yet fully understood, this phenomenon has become known as sleep-dependent memory consolidation (Maquet 2001; Stickgold 2005; Walker and Stickgold 2006).

Orexin peptides (orexin-A [or hypocretin-1] and orexin-B [or hypocretin-2]) play a primary role in regulating sleep and alertness (reviewed in de Lecea and Sutcliffe 2005; Kilduff and Peyron 2000; Ohno and Sakurai 2008; Saper et al. 2005), which has direct functional implications for diverse brain functions including learning and memory (Akbari et al. 2008; Akbari et al. 2006, 2007; Aou et al. 2003; Jaeger et al. 2002; Smith and Pang 2005; Wayner et al. 2004). Orexin neurons project to various brain regions including the basal forebrain (Peyron et al. 1998), an area implicated in the regulation of wakefulness and cortical desynchronization (Jones 2004), which contains cholinergic neurons that are active during waking and REM sleep (Lee et al. 2005). The cholinergic system is well known for its role in learning and memory, while its degeneration - as occurs in elderly people and patients with Alzheimer's disease - correlates with cognitive decline (Perry et al. 1981).

Almorexant is a dual orexin receptor antagonist, which reversibly blocks signaling of orexin-A and orexin-B peptides. Oral administration of almorexant elicits somnolence without cataplexy in healthy rats, dogs, and humans when 
given during the active phase of their circadian cycle (Brisbare-Roch et al. 2007). In the rat, almorexant maintains the natural architecture of sleep by decreasing active wakefulness and increasing the time spent in REM and NREM sleep in physiological proportion. This is in contrast to benzodiazepine receptor agonists, which tend to increase the time spent in quiet wakefulness and NREM sleep while shortening the time spent in REM sleep (Brisbare-Roch et al. 2007). The sleep state elicited by almorexant is easily surmountable and can be maintained upon repeated administration (Brisbare-Roch et al. 2007).

Here, we investigated the impact of high doses of almorexant on cognitive function in rodents using (1) a Morris water maze (MWM) spatial navigation (reference memory) task (Morris 1981, 1984), which requires rats to learn how to locate a water-submerged escape platform with the help of extra-maze cues and (2) a test of passive avoidance (PA) learning, which requires rats to suppress a specific behavior in order to avoid an aversive stimulus (for a review, see Byrne 2003). Studies using virtual versions of the MWM task in humans support the assumption that spatial learning in rodents and humans involves similar principles (Hamilton et al. 2002). Both spatial navigation in the MWM task and PA learning are well known to be sensitive to pharmacological manipulations of the cholinergic system (Lindner et al. 2006; Riekkinen et al. 1995, 1996; Whishaw 1985), and we used the muscarinic cholinergic receptor antagonist scopolamine as a reference drug to impair memory.

\section{Materials and methods}

\section{Subjects}

Each MWM experiment was conducted on 24 male LongEvans rats (Crl:LE, Charles River, Raleigh, NC, USA) of 1618 weeks of age. Sixty-four male Wistar rats (RccHan:WIST, Harlan Laboratories, Netherlands) of 12 weeks of age were tested on a PA task.

All rats were maintained in groups of four in a temperature-and humidity-controlled room with a 12h light-dark cycle, and with food and water provided ad libitum. Experiments were carried out between 8 a.m. and 5 p.m.

Morris water maze

MWM testing followed established procedures (Morris 1981, 1984). A circular white pool (diameter $1.8 \mathrm{~m}$; height $60 \mathrm{~cm}$ ) was filled $40 \mathrm{~cm}$ deep with water that was maintained at $26 \pm$ $1{ }^{\circ} \mathrm{C}$ and made opaque by the addition of an opacifier (an aqueous acrylic emulsion; Acusol, Rohm \& Haas, Landskrona, Sweden). A white plastic escape platform (diameter
$11 \mathrm{~cm}$ ) was placed in the center of one of the four equal-area quadrants of the pool.

In "cued" trials, the escape platform extended $1.5 \mathrm{~cm}$ above the water surface and the border of the escape platform was colored black to increase visibility. In "uncued" (referring to proximal cues) trials, the escape platform was submerged $1.5 \mathrm{~cm}$ below the water surface. In "probe" trials, no escape platform was present. The pool was surrounded by large, dissimilar, distal visual cues placed on the walls to assist allocentric orientation and spatial navigation. During trials, behavioral variables including the latency to climb the escape platform (escape latency), swim speed (average velocity), number of completed circles swum (pool circlings), and time spent by the rat in the water maze quadrants (time in quadrant) were measured using a video tracking system (HVS Image, Buckingham, UK). Time during which rats were not actively swimming (i.e., floating) was excluded from the calculation of velocity.

Each cued or uncued trial began by gently releasing a rat into the water facing the wall of the pool and ended when the rat climbed the escape platform or after a cut-off of $60 \mathrm{~s}$, whichever came first. When the rat did not reach the escape platform within $60 \mathrm{~s}$, the experimenter guided the rat to the escape platform. After completion of a trial, rats were allowed to stay on the escape platform for approximately $15 \mathrm{~s}$ for spatial orientation. Rats were gently removed from the escape platform, dried with a towel and kept in holding cages. At the end of each day's spatial training, rats were returned to their home cages.

For a timeline of the experiments, see Fig. 1a. $72 \mathrm{~h}$ before the first uncued trial, rats performed a total of six cued trials, analyzed in two blocks of three trials denoted as $\mathrm{C} 1$ and $\mathrm{C} 2$; this allowed the animals to become familiar with the experimental set-up and procedures. Rats were subsequently trained for spatial navigation using 24 uncued acquisition trials, analyzed in eight daily blocks of three trials (U1-U4 in the first week and U5-U8 in the second week). $24 \pm 2 \mathrm{~h}$ after the first and second weeks' last uncued trial, rats were tested for memory retention in two 30 s probe trials ( $\mathrm{P} 1$ and $\mathrm{P} 2)$ in which no escape platform was present. The time spent by rats in the "target quadrant" (the pool quadrant that had contained the escape platform during the spatial training period) relative to the other quadrants was analyzed as a measure of memory retention. The last probe trial P2 was followed by two cued trials, analyzed in one trial block (C3); these trials served as control measures for motivation and sensorimotor function.

The escape platform location was varied across cued trials. In uncued trials, escape platform location was varied across subjects but kept constant for each rat. To prevent the possibility of egocentric orientation, the location of the rat's entry into the pool was varied across trials. During probe trials, in order to keep the distance between the location of a rat's entry into the pool and the previously trained escape platform 
a

\begin{tabular}{|c|c|c|c|c|c|c|c|c|c|c|c|c|c|c|c|}
\hline Day & 1 & 2 & 3 & 4 & 5 & 6 & 7 & 8 & 9 & 10 & 11 & 12 & 13 & 14 & 15 \\
\hline & & & & & 1 & & T & & & & & & & & \\
\hline Drug tre & & & & $\checkmark$ & $\checkmark$ & $\checkmark$ & $\checkmark$ & & & & $\checkmark$ & $\checkmark$ & $\checkmark$ & $\checkmark$ & $\checkmark$ \\
\hline Trial block & $\mathrm{C} 1+\mathrm{C}$ & & & U1 & U2 & U3 & U4 & P1 & & & U5 & U6 & U7 & U8 & $\mathrm{P} 2+\mathrm{C} 3$ \\
\hline
\end{tabular}
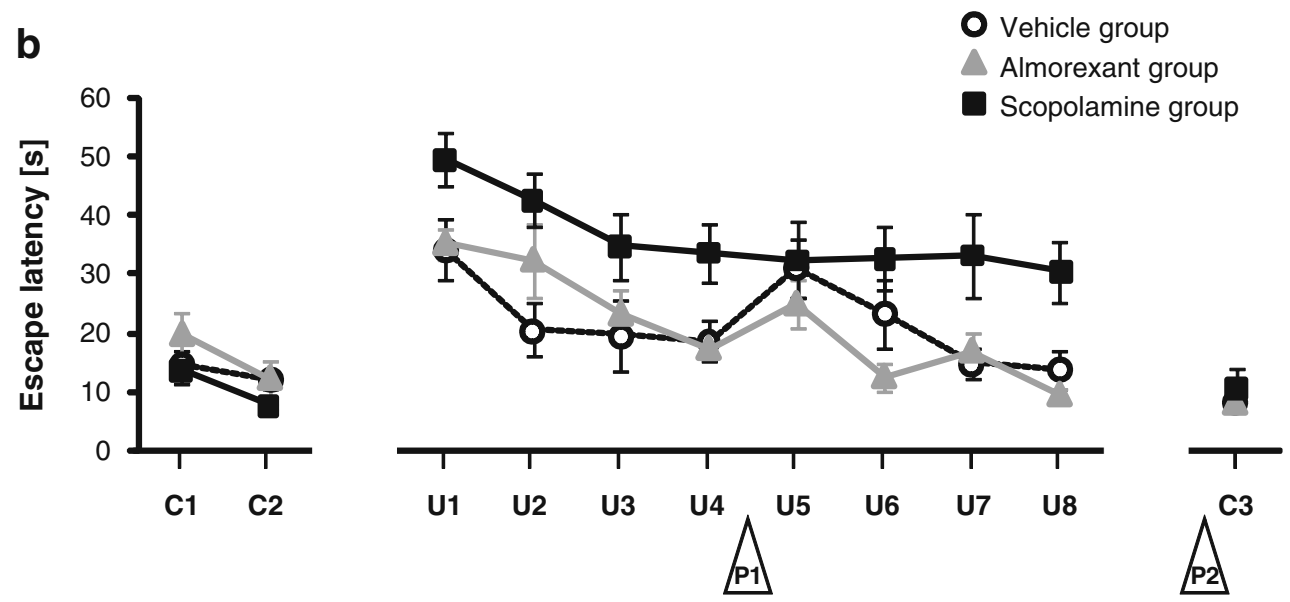

Fig. 1 a Experimental design illustrating the 15-day training and assessment protocol of Morris water maze (MWM) experiments. A tick mark indicates that rats received drug treatment on the respective day. b Spatial learning: latency in the MWM task to localize, approach, and climb an escape platform that was visible (cued trials, $\mathrm{C} 1-\mathrm{C} 2 ; \mathrm{C} 3$ ) or hidden (uncued trials, U1-U8). Rats were treated with vehicle only (open circles), almorexant (triangles), or scopolamine

location at a constant and maximal length, the position of entry into the pool was assigned to the quadrant opposite the target quadrant.

\section{Passive avoidance}

The "GEMINI" avoidance system with software part 68100006-C, equipped with the "AirStim" option (San Diego Instruments, San Diego, CA, USA), was used for tests of step-through type PA learning. One unit consisted of two compartments with inner dimensions of 25 (width) $\times 20$ (depth) $\times 17 \mathrm{~cm}$ (height). The walls were acrylic; the floor consisted of stainless steel grids (diameter $7 \mathrm{~mm}$ ), 14 per compartment, spaced $9 \mathrm{~mm}$ apart and aligned from front to back. An $8.5 \mathrm{~cm}$ (width) $\times 7.6 \mathrm{~cm}$ (height) rectangular opening at grid floor level connected both compartments. This opening could be closed by a stainless steel gate. The front wall of each compartment consisted of a door that could be opened to place a rat into or take it out of the compartment. The location of the rat was detected by infrared photoelectric beams, eight per compartment, positioned $13 \mathrm{~mm}$ above the grid floor. An LED house light was centered in the ceiling of each compartment, and supplied $650 \mathrm{~lx}$ at grid-floor level. (squares) before daily spatial training sessions. Trial blocks $\mathrm{C} 1-\mathrm{C} 2$ and U1-U8 include three trials per rat; trial block $\mathrm{C} 3$ includes two trials. Note that retention tests (probe trials P1 and P2, in open triangles) are depicted only to illustrate the order of trial blocks (for results on probe trials, see Fig. 2). Data are presented as mean \pm standard error of the mean with eight rats per treatment group

Air pulse stimulation was chosen as the aversive stimulus, and was delivered from two aluminum tubes (length $24.5 \mathrm{~cm}$, external diameter $6.5 \mathrm{~mm}$ ), one at the front and one at the back wall of each compartment, positioned $19 \mathrm{~mm}$ above the grid floor, and perforated at the vertical center with nine $2.5 \times$ $0.8 \mathrm{~mm}$ holes spaced $2.5 \mathrm{~cm}$ apart. Air was delivered to the system from a compressed air bottle at a static pressure of $379.2 \mathrm{kPa}$ (55 psi), which was set for each compartment separately using a regulator (Model R07, Norgren, Littleton, $\mathrm{CO}$, USA), yielding a pressure of $322.7 \mathrm{kPa}(46.8 \mathrm{psi})$ at presentation of air. Air was delivered at $3,160 \mathrm{~cm}^{3} / \mathrm{s}$ into the Tygon tubing (model AAG00007, Saint-Gobain Performance Plastics, Akron, OH, USA; inner diameter $3.2 \mathrm{~mm}$ ) that connected the regulator to the stimulus delivery tubes. When stimulation was initiated, three pulses of air were applied, each $0.5 \mathrm{~s}$ in duration and with $0.5 \mathrm{~s}$ separating each pulse (offset to onset). The actual pressure settings were sufficient to initiate a startle response.

The operant conditioning phase consisted of a single trial that began after a rat was gently placed into the right-hand compartment of a GEMINI unit (with front doors and gate closed, and lights turned off). After an acclimation period of $120 \mathrm{~s}$, the compartment in which the rat was located was illuminated and the gate to the left-hand, unilluminated 
compartment was opened. Step-through latency, i.e., the time until the rat completely stepped through into the left-hand compartment, was recorded. Once the rat stepped through, the gate was closed and, after a delay of $1 \mathrm{~s}$, air pulses were applied to the rat. The rat was allowed to stay in the left-hand compartment to associate the aversive event with the environment, after which it was returned to the home cage. Rats that did not step through within $600 \mathrm{~s}$ during the conditioning phase were excluded from the experiment.

The test phase, performed $24 \mathrm{~h}$ later, consisted of a single trial identical to the conditioning phase trial with the exception that no aversive stimulus was applied when the rat stepped through. An increase in step-through latency is considered to be an indicator of memory retention (i.e., conditioning success).
Drug treatment

Rats were assigned to one of four drug treatments:

1. Vehicle groups received oral treatment with a water-based solution containing $0.25 \%$ methyl cellulose (Methocel ${ }^{\circledR}$ MC, Fluka/Sigma-Aldrich Chemie GmbH, Steinheim, Germany) and intraperitoneal treatment with saline $(\mathrm{NaCl}$ $0.9 \%$, B. Braun Medical AG, Switzerland).

2. Almorexant groups received oral treatment with almorexant hydrochloride (Actelion Pharmaceuticals Ltd, Allschwil, Switzerland) in a water-based solution containing $0.25 \%$ methyl cellulose, at a dose of $300 \mathrm{mg} / \mathrm{kg}$ (calculated as almorexant free base), and intraperitoneal treatment with saline.
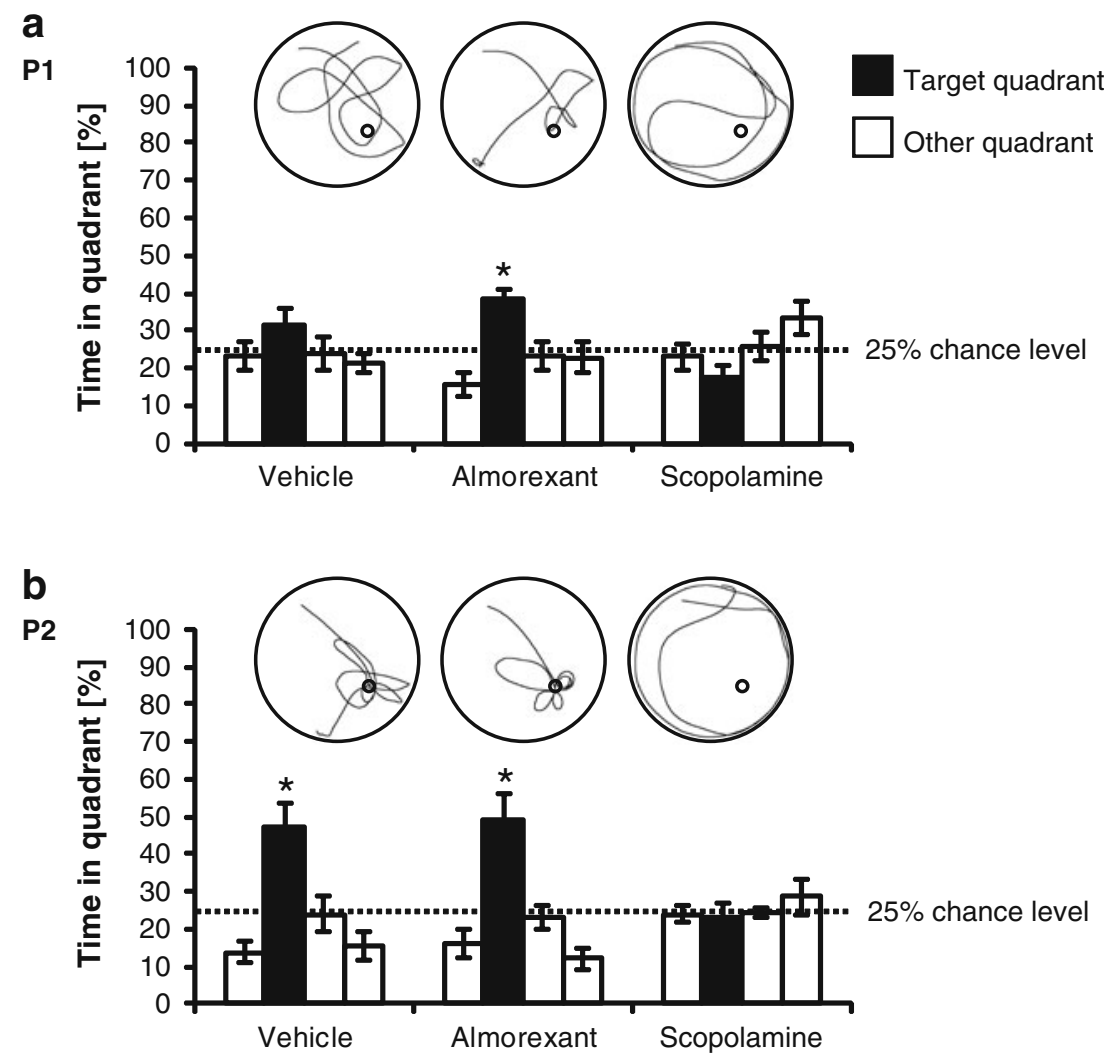

Fig. 2 Spatial memory retention: proportion of time spent by rats in the quadrants of the Morris water maze (MWM) during probe trials. After rats had been repeatedly trained on a MWM task under either vehicle, almorexant, or scopolamine treatment (see Fig. 1b), spatial memory retention tests were conducted $24 \pm 2 \mathrm{~h}$ after a the first week's (P1) and $\mathbf{b}$ the second week's (P2) last uncued trial block. No drug treatment was given at $\mathrm{P} 1$; drug treatment was given at $\mathrm{P} 2$ as on preceding days of spatial training (for a timeline, see Fig. 1a). Black bars represent the target quadrant (the quadrant that had contained the escape platform during the spatial training period); the bar on the left and the two bars on the right of each black bar represent the quadrants to the left, right, and opposite the target quadrant, respectively. Asterisks indicate that the proportion of time spent in the target quadrant was significantly above the chance rate of $25 \%$ $(p<0.05, T$ test for single means against a constant), i.e., spatial memory had been established. Data are presented as mean \pm standard error of the mean with eight rats per treatment group. Inserts show representative plots (top view) of individual swim paths of one rat for each treatment group and probe trial. In these examples, the start location was in the north-west quadrant of the pool, and the platform had formerly been located in the south-east quadrant of the pool (indicated by small circle for illustration purposes only; note that no platform was present in probe trials) 
3. Scopolamine groups received oral treatment with a waterbased solution containing $0.25 \%$ methyl cellulose and intraperitoneal treatment with scopolamine hydrobromide trihydrate (Sigma-Aldrich Chemie GmbH, Steinheim, Germany) in saline, at a scopolamine dose of $0.8 \mathrm{mg} / \mathrm{kg}$.

4. Combination (combo) groups received oral treatment with almorexant hydrochloride in a water-based solution containing $0.25 \%$ methyl cellulose, at an almorexant dose of $300 \mathrm{mg} / \mathrm{kg}$, and intraperitoneal treatment with scopolamine hydrobromide trihydrate in saline, at a scopolamine dose of $0.8 \mathrm{mg} / \mathrm{kg}$.

The first MWM experiment included a vehicle group, an almorexant group, and a scopolamine group, whereas the follow-up experiment included an almorexant group, a combo group, and a scopolamine group. The PA experiment included all four treatments.

In MWM experiments, treatment was given on every day of spatial training and on the day of the second probe trial P2; in order to control for state dependency, no treatment was given on the day of the first probe trial P1 (Fig. 1a). In the PA experiment, treatment was given once before the acquisition trial.

All treatments were administered with $5 \mathrm{ml} / \mathrm{kg}$. In all experiments, treatments were administered orally and intraperitoneally 60 and $30 \mathrm{~min}$, respectively, before task perfor- mance based on the pharmacokinetic and pharmacodynamic properties of almorexant (Brisbare-Roch et al. 2007) and published practice for scopolamine (e.g., McNamara and Skelton 1992; Riekkinen et al. 1996).

\section{Statistical analyses}

In order to determine statistically significant differences in escape latency, average velocity, and pool circlings in the MWM test, an analysis of variance (repeated measures twoway analysis of variance (ANOVA) for $\mathrm{C} 1-\mathrm{C} 2$, and $\mathrm{U} 1-\mathrm{U} 8$; one-way ANOVA for C3) was employed. A $T$ test comparing single means against a constant was used to determine if the proportion of time spent in the target quadrant of the MWM was significantly different from the chance rate of $25 \%$.

A Wilcoxon-matched pairs test was employed for the PA test to determine statistically significant intra-individual differences in step-through latency between the conditioning and test phases. A Mann-Whitney test was employed to determine significant differences in step-through latency between drug treatment groups.

Statistical testing was performed using statistical software (Statistica, version 6, StatSoft Inc., Tulsa, OK, USA; and Prism, version 5.01, GraphPad Software, Inc., USA) with differences considered statistically significant at $p<$ 0.05 . Data are presented as mean \pm standard error of the
Fig. 3 Spatial memory retention: proportion of time spent by rats in the quadrants of the Morris water maze during probe trials. See legend of Fig. 2 for details with exception of the following: rats were treated with either almorexant alone, both almorexant and scopolamine ("combo"), or scopolamine alone. In the examples of individual swim paths, the start location was in the south-west quadrant of the pool, and the platform had formerly been located in the north-east quadrant of the pool
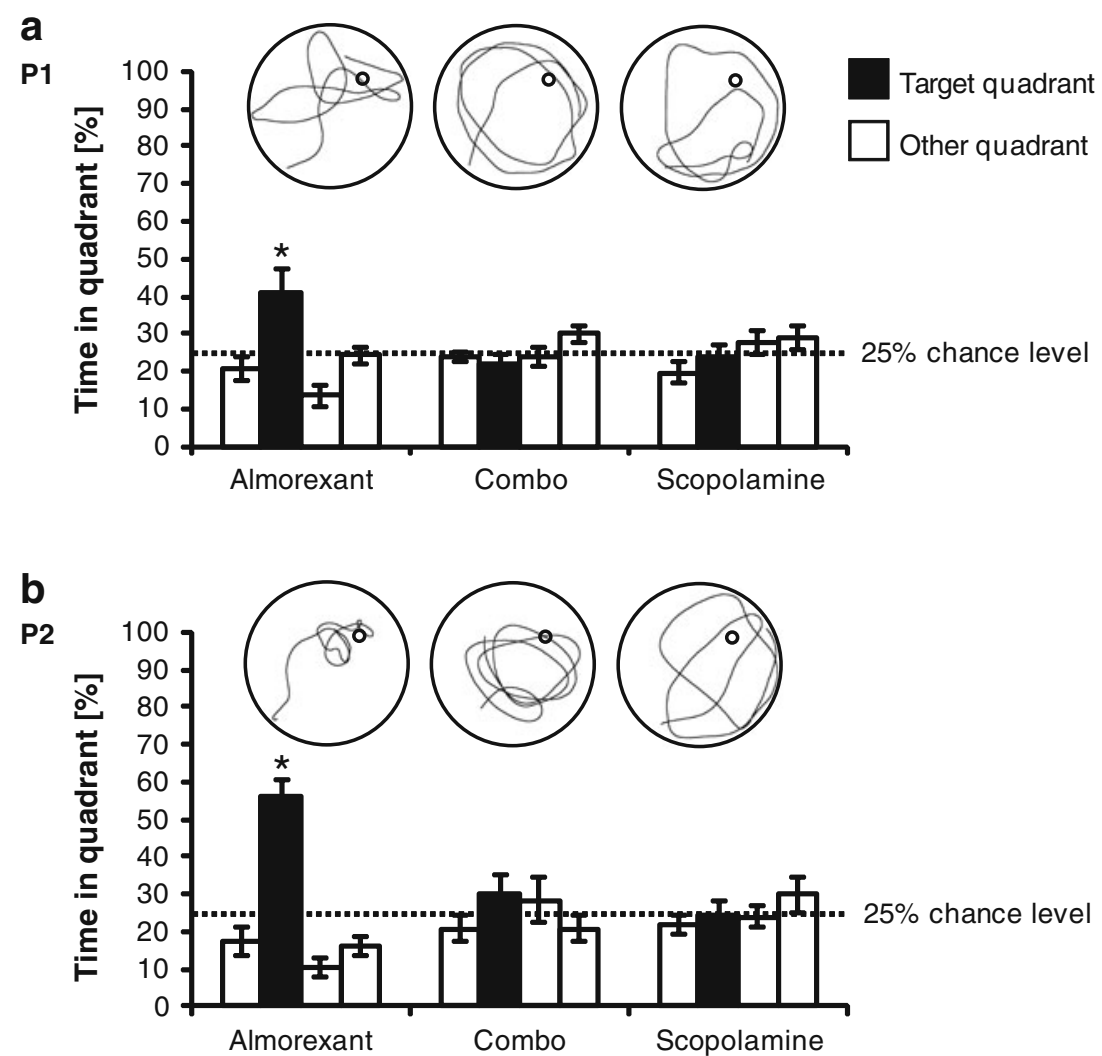
mean with eight (MWM) or 14-16 (PA) rats per treatment group.

\section{Results}

\section{Spatial learning in MWM}

Latency to localize, approach, and climb the escape platform in cued and uncued trials was assessed using a 15-day training and assessment protocol (Fig. 1a). Rats improved escape latency over time in both cued $(\mathrm{C} 1-\mathrm{C} 2, F(1,21)=8.2, p<0.01)$ and uncued trials (U1-U8, $F(7,147)=8.7, p<0.001)$ (Fig. 1b). Thus, rats were able to improve task performance over time independently of whether the platform was visible. No treatment effect on escape latency was found for cued trials $(\mathrm{C} 1-\mathrm{C} 2, F(2,21)=2.0, p=0.2 ; \mathrm{C} 3, \quad F(2,21)=0.5, p=0.6)$ (Fig. 1b), and no statistically significant interaction was observed between treatment and time for initial cued trials $(\mathrm{C} 1-\mathrm{C} 2, F(2,21)=0.5, p=0.6)$ (Fig. 1b), suggesting that treatment groups did not differ in their ability to solve the cued task variant.

During uncued trials, however, treatment groups differed $(\mathrm{U} 1-\mathrm{U} 8, F(2,21)=9.4, p<0.01)$ (Fig. 1b). The escape latency of scopolamine-treated rats, although it decreased over time, was higher compared with latencies of both of the other treatment groups throughout the entire time course. The analysis consistently failed to demonstrate a statistically significant interaction between treatment and time (U1-U8, $F(14,147)=1.1, p=0.4$ ) (Fig. 1b). In contrast to scopolaminetreated rats, both vehicle - and almorexant-treated rats successfully learned the spatial task; after 8 days of spatial training, they attained escape latencies similar to those observed in cued trials.

\section{Spatial memory retention in MWM}

Spatial memory retention was considered established when the proportion of time spent in the target quadrant during probe trials was significantly above the chance rate of $25 \%$.

In probe trial P1 (Fig. 2a) conducted $24 \pm 2 \mathrm{~h}$ after the first week's last uncued trial block (under no drug treatment), only almorexant-treated rats had established spatial memory $(T=4.9$, $d f=7, p<0.01$ ), whereas vehicle - and scopolamine-treated rats failed to establish spatial memory (vehicle group, $T=1.7, d f=7$, $p=0.1$; scopolamine group, $T=-2.4, d f=7, p=0.05$ ).

In probe trial $\mathrm{P} 2$ (Fig. $2 \mathrm{~b}$ ) conducted $24 \pm 2 \mathrm{~h}$ after the second week's last uncued trial block (under drug treatment), both vehicle-and almorexant-treated rats were found to have established spatial memory (vehicle group, $T=3.3, d f=7, p<0.05$; almorexant group, $T=3.3, d f=7, p<$ $0.05)$, whereas the scopolamine group failed to establish spatial memory $(T=-0.5, d f=7, p=0.6)$.
In a follow-up MWM experiment, the effect of the combination of almorexant and scopolamine was tested. Here, we only report results on spatial memory retention. In probe trials P1 and P2 (Fig. 3), only almorexant-treated rats had established spatial memory (P1, $T=2.7, d f=7, p=0.03$; P2, $T=7.7, d f=7, p<0.001)$, whereas combo-and scopolamine-treated rats failed to establish spatial memory (combo group, $\mathrm{P} 1, T=-1.1, d f=7, p=0.3, \mathrm{P} 2, T=1.0, d f=7$, $p=0.3$; scopolamine group, $\mathrm{P} 1, T=-0.3, d f=7, p=0.8, \mathrm{P} 2$, $T=-0.1, d f=7, p=0.9)$.

\section{Control measures in MWM}

To exclude the possibility that disturbances in sensorimotor function or motivation may have interfered with testing, we analyzed swim velocity and number of pool circlings. For initial cued trials, no statistically significant difference was found in either average velocity $(\mathrm{C} 1-\mathrm{C} 2$, $F(2,21)=1.2, p=0.3$ ) (Fig. $4 \mathrm{a}$ ) or pool circlings (C1-C2, $F$ $(2,21)=0.3, p=0.7) \quad($ Fig. $4 \mathrm{~b}$ ) across treatment groups, suggesting that treatment groups were comparable at the beginning of the experiment. As rats learned over time to escape onto the visible platform, pool circlings remained unchanged $(\mathrm{C} 1-\mathrm{C} 2, F(1,21)=1.8, p=0.2)$ (Fig. 4b) but average velocity increased $(\mathrm{C} 1-\mathrm{C} 2, F(1,21)=9.4, p<0.01)$ (Fig. 4a) to levels similar to those observed in vehicleand almorexant-treated rats at the beginning of spatial training.

During performance of the spatial task, treatment groups differed in both average velocity $(\mathrm{U} 1-\mathrm{U} 8, F(2,21)=11.5, p$ $<0.001$ ) (Fig. 4a) and pool circlings (U1-U8, $F(2,21)=$ 14.0, $p<0.001$ ) (Fig. 4b). Swimming in circles, as opposed to the navigation strategy in which rats learn to use spatial cues to localize the escape platform, may serve as an alternative behavioral strategy that increases the probability of hitting the submerged escape platform by chance. We observed that rats swimming in circles, often in combination with thigmotaxis, swam faster than rats appearing to employ the navigation strategy (data not shown). A consistently higher number of pool circlings and, possibly as result, consistently higher swim velocities were observed for scopolamine-treated rats compared with almorexant-or vehicle-treated rats. Time effects were observed for both average velocity $(\mathrm{U} 1-\mathrm{U} 8, F(7,147)=4.9, p<0.0001)$ (Fig. 4a) and pool circlings (U1-U8, $F(7,147)=3.2, p<$ 0.01) (Fig. 4b).

No interactions between treatment and time were observed for both average velocity and pool circlings (C1-C2, average velocity, $F(2,21)=0.2, p=0.8$, pool circlings, $F(2,21)=0.5, p=$ 0.6 ; U1-U8, average velocity, $F(14,147)=1.0 p=0.4$, pool circlings, $F(14,147)=0.6, p=0.9)$ (Fig. 4). In final cued trials, treatment affected average velocity $(\mathrm{C} 3, F(2,21)=10.4, p<$ 0.001 ) (Fig. 4a) but not pool circlings $(\mathrm{C} 3, F(2,21)=1.0, p=$ 
Fig. 4 a Swim velocity and b number of complete pool circlings. Times during which rats were not actively swimming (i.e., when floating) were excluded from the velocity calculations. For other details, see legend of Fig. 1

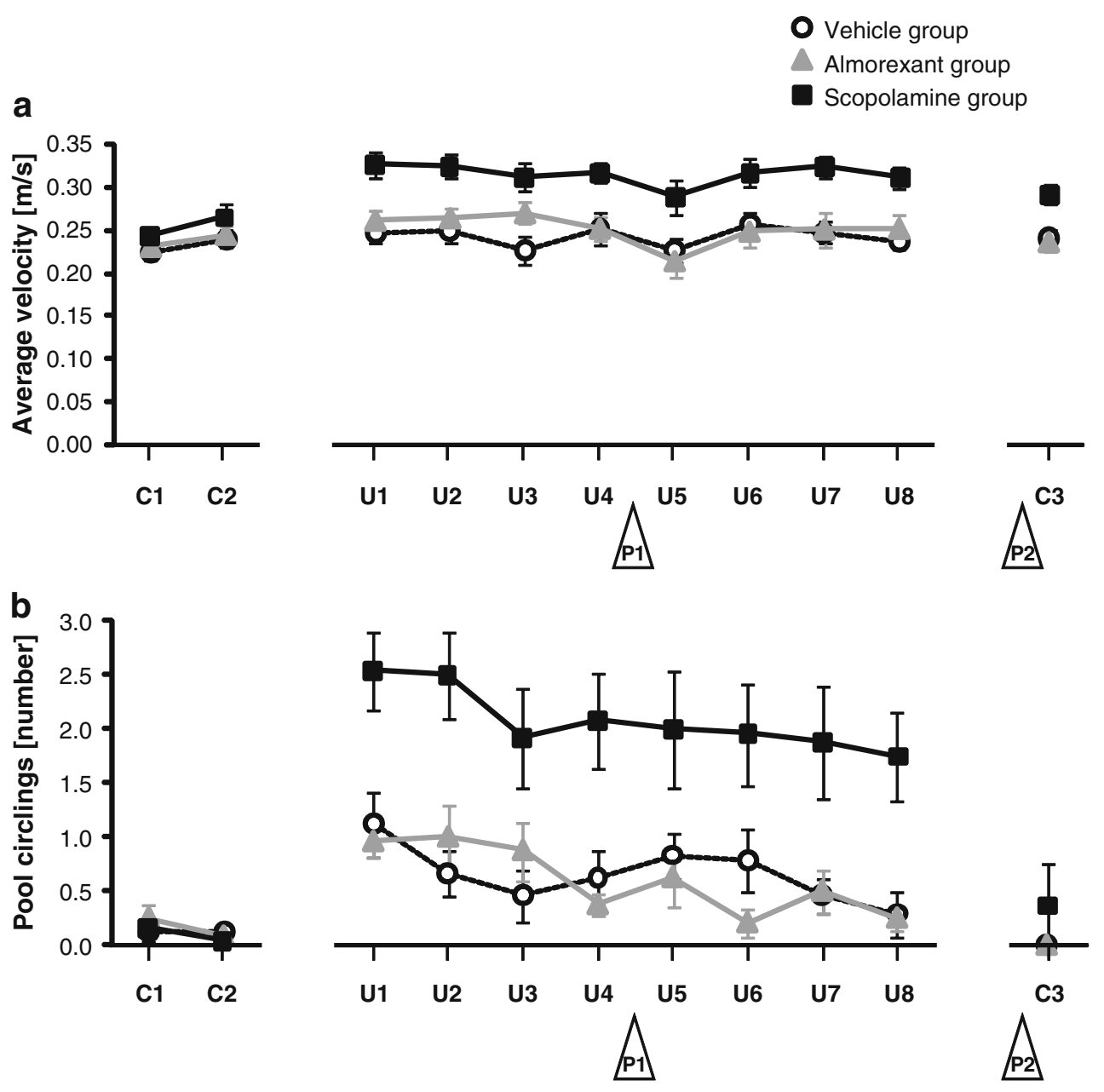

Vehicle group

$\begin{array}{llllllll}\text { U1 } & \text { U2 } & \text { U3 } & \text { U4 } & \text { U5 } & \text { U6 } & \text { U7 } & \text { U8 }\end{array}$

C3
0.4) (Fig. 4b); the presence of a visual cue thus diminished pool circlings but did not return average velocity to normal values in scopolamine-treated rats.

\section{Avoidance memory retention in PA}

Memory retention was considered established when step-through latency during the conditioning phase was statistically different from step-through latency during the test phase. Memory retention was intact in both vehicle-treated rats $(p<0.05)$ and almorexant-treated rats $(p<0.01)$ but this was not the case for either scopolamine $-(p=0.6)$ or combo-treated rats $(p=0.1)$.

In the test phase, almorexant-treated rats did not differ in step-through latency from vehicle-treated rats $(p=0.2)$ but both scopolamine $-(p<0.05)$ and combo-treated rats $(p<0.01)$ did (Fig. 5).

\section{Discussion}

Our results show that rats treated with repeated doses of almorexant are fully capable of establishing spatial memory in a MWM spatial navigation task that is sensitive to agents which impair hippocampus-dependent declarative learning. After 4 days of spatial training, only almorexant-treated rats had established spatial memory; after 8 days of spatial training, both vehicle - and almorexant-treated rats demonstrated spatial memory retention. In contrast, rats treated with scopolamine failed to learn the task and did not show spatial memory retention after either 4 or 8 days of spatial training. The result of the first memory retention test after 4 days of spatial training suggests that almorexant facilitated memory encoding and/or consolidation processes during the spatial acquisition phase and thus may have accelerated establishment of spatial memory. However, almorexant did not ameliorate the scopolamine-induced impairment of spatial memory. The fact that rats in the almorexant group demonstrated memory retention under undrugged conditions during the first memory retention test conducted after 4 days of spatial training suggests that the memory retrieval process was not state dependent, i.e., rats did not need to be under the influence of almorexant to retrieve memory. The results of the second memory retention test conducted under drug influence after 8 days 


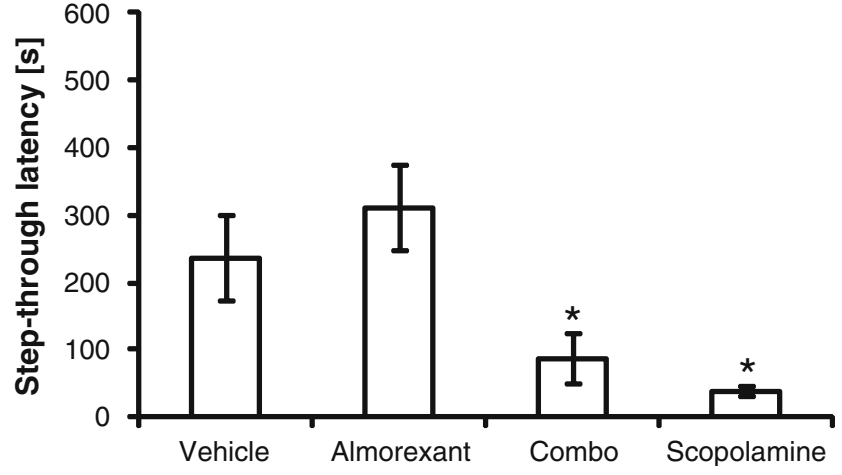

Fig. 5 Test of passive avoidance learning: an avoidance memory retention test was conducted $24 \mathrm{~h}$ after rats had been trained once on a task of passive avoidance learning under either vehicle, almorexant, combo (both almorexant and scopolamine), or scopolamine treatment. Avoidance memory retention was intact in both vehicle and almorexant-treated rats (see text). Asterisks indicate step-through latency of the respective drug treatment group was statistically different from step-through latency of the vehicle group. Data are presented as mean \pm standard error of the mean with $14-16$ rats per treatment group

of spatial training suggest that almorexant does not interfere with retrieval of spatial memory.

Both vehicle-treated rats and almorexant-treated rats were able to learn the passive avoidance task; in contrast, scopolamine-treated rats and rats treated with the combination of almorexant and scopolamine were not able to learn the task. Thus rats treated with almorexant are fully capable of avoidance learning in a PA task that is sensitive to agents which impair associative learning, which is in line with our MWM findings.

Almorexant increases the time spent in NREM and REM sleep. Over a $12 \mathrm{~h}$ night, rats treated with almorexant at a dose of $300 \mathrm{mg} / \mathrm{kg}$ spent $41.1 \%$ of the time in NREM sleep and $7.9 \%$ in REM sleep versus $31.8 \%$ in NREM sleep and $4.5 \%$ in REM sleep for vehicle-treated rats (Brisbare-Roch et al. 2007). This increase in the proportion of NREM and REM sleep may contribute to beneficial effects on learning and memory. Although controversially discussed (Genzel et al. 2009), evidence has accumulated over the last decade in favor of sleep-dependent learning and memory processes (reviewed in Born et al. 2006; Stickgold 2005; Stickgold and Walker 2005; Walker and Stickgold 2006; Wayner et al. 2004). Functional imaging data from healthy human subjects indicates that sleep is implicated in the consolidation of declarative memory (Gais et al. 2007; Sterpenich et al. 2007). It has been suggested that NREM sleep is crucial for declarative memory processes (Plihal and Born 1999; Rasch et al. 2009). On the other hand, REM sleep might be particularly beneficial for procedural memory (Rasch et al. 2009; Smith 1995).

The cholinergic system in the basal forebrain, hippocampus, amygdala, and neocortex is well known to be implicated in memory processing (Everitt and Robbins 1997) and high cholinergic tone is considered a prerequisite for efficient encoding of memories during wakefulness (Bartus et al. 1982; Rasch et al. 2006). The muscarinic cholinergic receptor antagonist scopolamine has been widely used to induce the cognitive deficits associated with cholinergic hypofunction. Pharmacological blockade of central muscarinic receptors has been reported to induce short-term amnestic effects for a variety of cognitive tasks in normal human subjects, nonhuman primates, and rodents (McNamara and Skelton 1992, and references therein).

Our results are in line with literature reports indicating that cholinergic antagonists impair spatial learning in the MWM but not cue learning (Whishaw 1985). The scopolamine amnesia model has been criticized for non-mnemonic side effects (Beiko et al. 1997; Terry 2006), which might be held responsible for the increased pool circlings and swim velocity that we observed with scopolamine treatment. However, scopolamine-induced spatial memory deficits have been demonstrated even under conditions that prevented drug side effects by utilizing a novel delayed matching to position water maze task (von Linstow Roloff et al. 2007).

In contrast to the deficits induced by cholinergic antagonists, which appear to be qualitatively similar to those induced by benzodiazepine receptor agonists (McNamara and Skelton 1992), almorexant did not impair acquisition of spatial learning or other abilities required to solve the MWM task despite the sleep state that almorexant induces in rats at the dose used. Notably, almorexant had no detrimental effect on task performance. Almorexant promotes a state of sleep that, if needed, is immediately and fully reversible (Brisbare-Roch et al. 2007), although in nocturnal animals, such as rats, the hypnotic effect of high-dose almorexant given during the day is lower than its effect at night (Brisbare-Roch et al. 2007). In our MWM experiments, cued trials served as a control for sensorimotor function and motivation of the rats, since factors such as impaired vision (which may prevent rats from using proximal or distal visual cues), disturbed motor performance (which may lead to an abnormal swim velocity and impair the ability to climb the platform), or a lack of motivation (which may lead to unusual amounts of floating), would compromise both cued and uncued trials. The finding that almorexant had no effect on escape latency in control trials suggests that the effects observed during spatial training are truly related to learning and memory processes.

Orexinergic innervation of both cholinergic and GABAergic septohippocampal neurons has been suggested as a mechanism by which the orexin system modulates spatial learning and memory (Smith and Pang 2005).

Interesting but contradictory results have been reported on the MWM task with (1) intracerebroventricular infusion of orexin-A (Aou et al. 2003), (2) lesions in the septohippocampal system induced by orexin-saporin, a toxin targeting orexin-B receptors (Smith and Pang 2005), 
and (3) infusion of the $\mathrm{OX}_{1}$ receptor antagonist SB-334867 into hippocampal CA1 or dentate gyrus (Akbari et al. 2006, 2007); and on the PA task with (1) intracerebroventricular infusion of orexin-A (Jaeger et al. 2002; Telegdy and Adamik 2002), and (2) infusion of SB-334867 into hippocampal CA1 or dentate gyrus (Akbari et al. 2008). These studies employed methodologies that differed from our experiments with respect to: the design of the MWM protocol (e.g., single- versus multiple-day schedules); the design of the PA protocol (e.g., step-down versus stepthrough type of PA, electric food shock versus air pulse stimulation); efficacy and selectivity of the test substances for orexin receptors (agonist versus $\mathrm{OX}_{1}$ or dual $\mathrm{OX}_{1} / \mathrm{OX}_{2}$ antagonists); route of administration (local infusion versus oral treatment); and, in some cases, in the strain of rats tested (albino versus pigmented). Despite the fact that the effects reported were heterogeneous and also contradictory, taken together with our findings, they clearly indicate an involvement of the orexinergic system in learning and memory. We report here that rats treated with almorexant are fully capable of both spatial and avoidance learning. Whether almorexant could indirectly elicit a beneficial effect on cognitive functions via its sleep-promoting properties remains to be further investigated.

Acknowledgments The authors are full-time employees at Actelion Pharmaceuticals Ltd, the sponsor of this study. The technical contribution from many scientists at Actelion's Research \& Development Center, Allschwil, Switzerland, is greatly acknowledged. Special thanks to Dr Catherine Brisbare-Roch and Dr Michel Steiner for fruitful discussions on the neurobiology of orexins in cognitive processes.

Experimental procedures used in this study received regulatory veterinary approval and followed international guidelines, the Principles of Laboratory Animal Care (Guidelines for the Care and Use of Mammals in Neuroscience and Behavioral Research, US National Research Council), and Swiss federal regulations on animal experimentation.

Open Access This article is distributed under the terms of the Creative Commons Attribution Noncommercial License which permits any noncommercial use, distribution, and reproduction in any medium, provided the original author(s) and source are credited.

\section{References}

Akbari E, Naghdi N, Motamedi F (2006) Functional inactivation of orexin 1 receptors in CA1 region impairs acquisition, consolidation and retrieval in Morris water maze task. Behav Brain Res $173: 47-52$

Akbari E, Naghdi N, Motamedi F (2007) The selective orexin 1 receptor antagonist SB-334867-A impairs acquisition and consolidation but not retrieval of spatial memory in Morris water maze. Peptides 28:650-656

Akbari E, Motamedi F, Naghdi N, Noorbakhshnia M (2008) The effect of antagonization of orexin 1 receptors in CA1 and dentate gyrus regions on memory processing in passive avoidance task. Behav Brain Res 187:172-177
Aou S, Li XL, Li AJ, Oomura Y, Shiraishi T, Sasaki K, Imamura T, Wayner MJ (2003) Orexin-A (hypocretin-1) impairs Morris water maze performance and CA1-Schaffer collateral long-term potentiation in rats. Neuroscience 119:1221-1228

Bartus RT, Dean RL 3rd, Beer B, Lippa AS (1982) The cholinergic hypothesis of geriatric memory dysfunction. Science 217:408-414

Beiko J, Candusso L, Cain DP (1997) The effect of nonspatial water maze pretraining in rats subjected to serotonin depletion and muscarinic receptor antagonism: a detailed behavioural assessment of spatial performance. Behav Brain Res 88:201-211

Born J, Rasch B, Gais S (2006) Sleep to remember. Neuroscientist $12: 410-424$

Brisbare-Roch C, Dingemanse J, Koberstein R, Hoever P, Aissaoui H, Flores S, Mueller C, Nayler O, van Gerven J, de Haas SL, Hess P, Qiu C, Buchmann S, Scherz M, Weller T, Fischli W, Clozel M, Jenck F (2007) Promotion of sleep by targeting the orexin system in rats, dogs and humans. Nat Med 13:150-155

Byrne J (ed) (2003) Learning \& memory. Thomson/Gale, New York

de Lecea L, Sutcliffe JG (2005) The hypocretins and sleep. FEBS J 272:5675-5688

Everitt BJ, Robbins TW (1997) Central cholinergic systems and cognition. Annu Rev Psychol 48:649-684

Gais S, Albouy G, Boly M, Dang-Vu TT, Darsaud A, Desseilles M, Rauchs G, Schabus M, Sterpenich V, Vandewalle G, Maquet P, Peigneux P (2007) Sleep transforms the cerebral trace of declarative memories. Proc Natl Acad Sci USA 104:1877818783

Genzel L, Dresler M, Wehrle R, Grozinger M, Steiger A (2009) Slow wave sleep and REM sleep awakenings do not affect sleep dependent memory consolidation. Sleep 32:302-310

Hamilton DA, Driscoll I, Sutherland RJ (2002) Human place learning in a virtual Morris water task: some important constraints on the flexibility of place navigation. Behav Brain Res 129:159-170

Jaeger LB, Farr SA, Banks WA, Morley JE (2002) Effects of orexin-A on memory processing. Peptides 23:1683-1688

Jones BE (2004) Activity, modulation and role of basal forebrain cholinergic neurons innervating the cerebral cortex. Prog Brain Res 145:157-169

Kilduff TS, Peyron C (2000) The hypocretin/orexin ligand-receptor system: implications for sleep and sleep disorders. Trends Neurosci 23:359-365

Lee MG, Hassani OK, Alonso A, Jones BE (2005) Cholinergic basal forebrain neurons burst with theta during waking and paradoxical sleep. J Neurosci 25:4365-4369

Lindner MD, Hogan JB, Hodges DB Jr, Orie AF, Chen P, Corsa JA, Leet JE, Gillman KW, Rose GM, Jones KM, Gribkoff VK (2006) Donepezil primarily attenuates scopolamine-induced deficits in psychomotor function, with moderate effects on simple conditioning and attention, and small effects on working memory and spatial mapping. Psychopharmacology (Berl) 188:629-640

Maquet P (2001) The role of sleep in learning and memory. Science 294:1048-1052

McNamara RK, Skelton RW (1992) Assessment of a cholinergic contribution to chlordiazepoxide-induced deficits of place learning in the Morris water maze. Pharmacol Biochem Behav 41:529-538

Morris RGM (1981) Spatial localisation does not depend on the presence of local cues. Learn Mem 12:234-260

Morris RGM (1984) Developments of a water-maze procedure for studying spatial learning in the rat. J Neurosci Methods 11:47-60

Ohno K, Sakurai T (2008) Orexin neuronal circuitry: role in the regulation of sleep and wakefulness. Front Neuroendocrinol 29:70-87

Perry EK, Blessed G, Tomlinson BE, Perry RH, Crow TJ, Cross AJ, Dockray GJ, Dimaline R, Arregui A (1981) Neurochemical activities in human temporal lobe related to aging and Alzheimertype changes. Neurobiol Aging 2:251-256 
Peyron C, Tighe DK, van den Pol AN, de Lecea L, Heller HC, Sutcliffe JG, Kilduff TS (1998) Neurons containing hypocretin (orexin) project to multiple neuronal systems. J Neurosci 18:9996-10015

Plihal W, Born J (1999) Effects of early and late nocturnal sleep on priming and spatial memory. Psychophysiology 36:571-582

Rasch BH, Born J, Gais S (2006) Combined blockade of cholinergic receptors shifts the brain from stimulus encoding to memory consolidation. J Cogn Neurosci 18:793-802

Rasch B, Gais S, Born J (2009) Impaired off-line consolidation of motor memories after combined blockade of cholinergic receptors during REM sleep-rich sleep. Neuropsychopharmacology 34:1843-1853

Riekkinen P Jr, Kuitunen J, Riekkinen M (1995) Effects of scopolamine infusions into the anterior and posterior cingulate on passive avoidance and water maze navigation. Brain Res 685:46-54

Riekkinen P Jr, Schmidt B, Stefanski R, Kuitunen J, Riekkinen M (1996) Metrifonate improves spatial navigation and avoidance behavior in scopolamine-treated, medial septum-lesioned and aged rats. Eur J Pharmacol 309:121-130

Saper CB, Scammell TE, Lu J (2005) Hypothalamic regulation of sleep and circadian rhythms. Nature 437:1257-1263

Smith C (1995) Sleep states and memory processes. Behav Brain Res 69:137-145

Smith HR, Pang KC (2005) Orexin-saporin lesions of the medial septum impair spatial memory. Neuroscience 132:261-271
Sterpenich V, Albouy G, Boly M, Vandewalle G, Darsaud A, Balteau E, Dang-Vu TT, Desseilles M, D'Argembeau A, Gais S, Rauchs G, Schabus M, Degueldre C, Luxen A, Collette F, Maquet P (2007) Sleep-related hippocampo-cortical interplay during emotional memory recollection. PLoS Biol 5:e282

Stickgold R (2005) Sleep-dependent memory consolidation. Nature 437:1272-1278

Stickgold R, Walker MP (2005) Sleep and memory: the ongoing debate. Sleep 28:1225-1227

Telegdy AV, Adamik A (2002) The action of orexin A on passive avoidance learning. Involvement of transmitters. Regul Pept 104:105-110

Terry AV Jr (2006) Muscarinic receptor antagonists in rats. In: Levin ED, Buccafusco JJ (eds) Animal models of cognitive impairment (Frontiers In Neuroscience). CRC Press, Boca Raton, pp 5-20

von Linstow Roloff E, Harbaran D, Micheau J, Platt B, Riedel G (2007) Dissociation of cholinergic function in spatial and procedural learning in rats. Neuroscience 146:875-889

Walker MP, Stickgold R (2006) Sleep, memory, and plasticity. Annu Rev Psychol 57:139-166

Wayner MJ, Armstrong DL, Phelix CF, Oomura Y (2004) Orexin-A (Hypocretin-1) and leptin enhance LTP in the dentate gyrus of rats in vivo. Peptides 25:991-996

Whishaw IQ (1985) Cholinergic receptor blockade in the rat impairs locale but not taxon strategies for place navigation in a swimming pool. Behav Neurosci 99:979-1005 